\title{
The Design and Realization on Effectively Fire Tower Planning Based on MapGIS-TDE
}

\author{
Wenyou FAN, Xin MENG, Xiaojing LIU, Niaoniao HU \\ ${ }^{1}$ Faculty of Information Engineering, China University of Geosciences, Wuhan, China \\ ${ }^{2}$ Research Center for GIS software and Application Engineering, Ministry of Education \\ Email:mapsuv@126.com
}

\begin{abstract}
Fire-tower is effectively applied in forest fire prevention and commanding system, especially in fire monitor and position. After explaining the significance of scientific planning of fire-tower, this paper analyzes GIS`s functions in building forest fire prevention system. This paper uses case study method, which designs a model, fire tower planning and analysis, based on MapGIS platform. After that, it directs us how to realize these functions based on MapGIS-TDE which is a 3D platform belonged to MapGIS. This paper gives us scientific ways to fire tower planning in forest fire system which promotes informationization of forest fire prevention management.
\end{abstract}

Keywords: MapGIS-TDE, forest fire prevention, 3D, fire tower planning, visual analysis

\section{Introduction}

Forest fire prevention has already become a global question. Countries around the world put plenty of money in researching forest fire prevention technology to reduce loss in fire accident. Fire tower, cruise aircraft and satellite monitoring are three ways to monitor forest fire, but fire tower is the major tool for forest fire prevention in china so far.

Fire tower `s primary target is to find and report fire in time. To avoid blind spots in monitoring and provide more accurate fire information in limited time, scientific planning fire tower become very important. On the other hand, it will save a great deal of money if we can scientifically plan fire tower and maximize the actual performance of fire tower.

\section{System Introduction}

Location principle is primary for fire tower planning and analysis. Under the designed principle and powerful GIS spatial analysis capabilities, the fire tower can realize visual analysis functions, which will be explained in three layers specifically.

One is support layer which includes entire operational environment and core technologies. Fire tower`s visual analysis function mainly based on 3D information which is different from many others functions in forest fire prevention system. Fire tower visual analysis will lose effectiveness even without 3D information. We can conclude that 3D visual technology is the key.

The second layer is logic layer. When we build an ap- plication system, we have to make sure that technology is appropriate to actual work flow.

The third layer is interaction layer which is used by customers. When we designed this layer, we need to consider customer`s habits.

\section{System Design}

\subsection{General Program}

By fully considering these three layers, this paper proposed the overall design program, including data, model, and function shown as in Figure1,

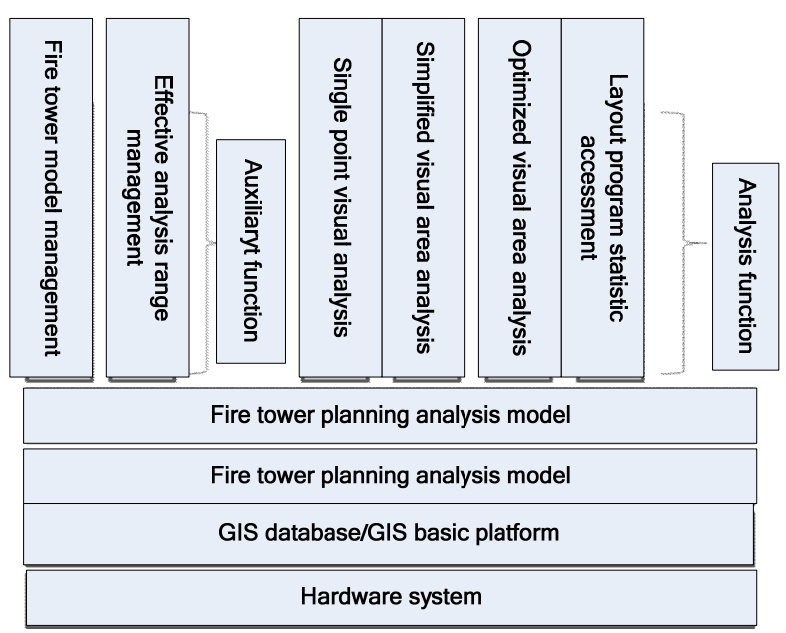

Figure 1. General structure 
Fire tower planning required at least two type data: first is digital elevation model (DEM) in planning area; the second type is specialized drawing and 3D model of fire tower.

Planning analysis model defined as an algorithm is to realize planning analysis function. We must consider two professional concepts based on purely geographic and spatial analysis: one concept is effective planning scope. We need to define analysis area, for a municipal forest fire prevention system are based on the city`s terrain map. Second is effective resolution range. The pyrotechnic devices which installed in fire tower such as (camera and supporting software) just like human eyes, which only can view a limited distance. Fire town can not monitor things beyond the distance which is the inevitable factor.

System functions design is based on data and model, combining with actual work flow.

\subsection{Design Application Functions}

There are two core parts in fire tower planning analysis: one is analysis, which calculated and showed varies of visible and invisible result and also the visible scope. The other is access which is to give quantitative suggestions for multi-location selection and layout program.

Based on the cores, we can realize system functions from four following aspects:

1) Analysis of single visible point

Single-point analysis is based on single observation tower, which research visible situation of any point within effective planning area. There are two results from that analysis: visible or invisible. Fire tower and target point will be connected and the results will be displayed in connected lines.

2) Simplify analysis of visible area

Visible area analysis also uses single fire tower as analyzing objective researching coverage of single fire tower within the effective scope. The analyzing results which included visible and invisible areas are distinguished by different colors, so we can compare with scopes of two kinds of areas very intuitively and qualitatively.

3) Optimizing analysis of visible area

It introduces fire tower resolution range factor from "Simplify analysis of visible area". All the area beyond the scope will be invisible and the result will be closer to the fact.

4) Layout program statistic and assessment

It analyzes multi fire tower. In addition to consider single fire tower, it also considers the overlapped area of the visible area, and finally quantitatively evaluates the solutions.

\section{Realization of Major Functions}

According to scheme of fire tower planning and analysis, the system adopts MapGIS K9 as basic platform and
MapGISK9-TDE as 3D platform which mainly uses C/S model. The following are simple function realization ideas.

\subsection{Single Point visual Analysis}

It has two steps:

1) get distinguishable distance of fire tower

In order to enhance system`s portability ability, system will take distinguishable distance as fire tower attribute. We can automatically obtain its attribute information when we choose analyzed objective.

Because fire tower is stored in 3D model, and its attribute query interface is slightly different with the general3D elements:

long flg = Get3DFeatuLayer(m_pGdataBase, "fire tower", Temp3DLayerInfo) \{\}

This interface includes three parameters: m_pGdataBase represents the geodatabase pointer in current operation; the "fire tower" string constants are the names of the 3D layers defined by database; Temp3DLayerInfo the 3D element layer information input after pointer visiting designed layer, which includes distinguishable distance information.

2) judge fire tower visibility

From the technology point of view, fire tower visibility can be abstracted as inter-perspective analyses of two points. The call interface can be defined as,

VisiableBe-

tweenTwoDot(start3Dot,end3Dot,visiableDist)

\{

//get the straight-line distance, if it beyond the distinguishable distance, then "invisible"

long distance = GetDistanceBe-

tweenTwoDot(tart3Dot,end3Dot);

if(distance $>$ visiableDist $)\{$ return false;

\}

//inter-perspective analyses of two points

bool flg = VisiableAnalyByTwoDot(start3Dot,end3Dot);

\}

The effective picture of single point visible analysis is shown in Figure 2:

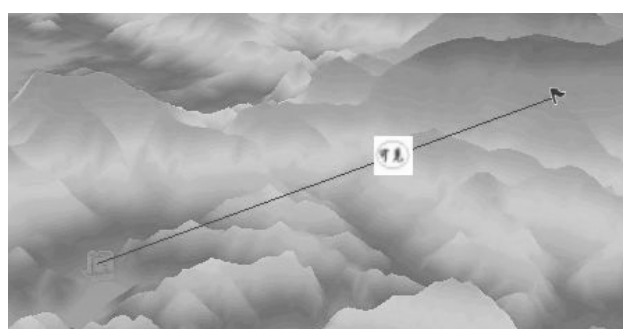

Figure 2. Single point visible analysis effective picture 


\subsection{Simplify Analysis of Visual Area}

Simplify analysis of visual area has three steps,

1) get effective analyzed scope

There are several methods for effective analyzed scope setting. Subjectively, manually set forest geological scope; objectively, take the pre-selected location as circle and observations distance as radius, since this method is interfered by lots of factors, which will not be the key point of this paper.

Obtain a valid analytical range is to read the predetermined range results, and pass to the following up process module. The interface is as follows:

Polygon polygon $=$ GetAnalyRange $1($ RegDotArr $)\{\}$

The interface only has one parameter that is the boundary string coordinates in effective range. After setting the scope, the boundary string coordinates can be stored in any visiting database table by string splicing or point by point record method. RegDotArr is defined as 3D coordinates object C3DotArr in MAPGIS-TDE. After convert the string coordinates to interfaces by definition, we can return to polygon region object which consistent with the effective analyzing scope.

2) fire tower visible area analysis

In fire tower visible area analysis, the effective scope analysis obtained in the previous step will be transferred to the following interface,

bool flg = VisibleAnalysysByPolygon(m_pGdataBase,3d Dot, polygon, visiableId,invisiableId)

m_pGdataBase also represents geodatabase pointer in current operation. 3dDot is a 3D point object, which is used to indicate the location of fire tower. After that, the analyzed results will be stored in geodatabase in raster image. visiableId and invisiableId are the output image identification.

3) Visualized visible area analyzed result

After analysis, the results need to be displayed for user identify. The interface is designed as follows,

bool flg = ShowVisibleAnalysysRlt(m_pGdataBase, visiableId, invisiableId) \{\}

VisiableId and InvisiableId are the analyzed resulting images stored in geographic database. This method is to add image into 3D scene through geodatabase pointer and image ID.

\subsection{Optimize Visible Area Analysis}

The realization method is quite the same with the above. It only adds one process in effective analysis scope.

1) get effective analysis scope

Optimized analysis of the visible field needs to take the fire tower distinguish parameter into practice, while the camera in fire tower can adjust the horizontal and vertical viewing angle in both directions. The system design takes the fire tower center as circle and the distinguish distance as radius to form a circular region, that is, the fire tower visible range. Then overlap the previous set area. The intersection regions can be seen as the effective range of optimized visible field analysis. Shown as follows:

Polygon polygon $=$ GetAnalyRange2(RegDotArr,3d Dot,visiableDist)

\{

\{

//calculate fire tower visible area

Circle circle $=$ GetVisiableRange(3dDot,visiableDist $)$ //get planned analysis scope

Polygon polygon $=$ GetAnalyRange1 $($ RegDotArr $)$

//calculate the intersection of visible area and planning analysis ygon)

Polygon polygon $=$ GetCrossingRange $($ circle, pol-

\}

2) fire tower visible area analysis

Similar to the simplified visible area analysis of fire tower, call visible area analysis interface gets gird image analysis result. The difference is that, system will get visible area in this area, not only take the left part as invisible area. It takes invisible area expands to "planning analysis scope" and shaded areas are not visible. As shown in Figure 3.

3) display the analysis results of visible area

The last step analysis result display effect is as follows. We can see effective picture from figure 4 that the boundary of red outline is circular, and the left blue area is invisible, not only the blue in the circular are invisible.

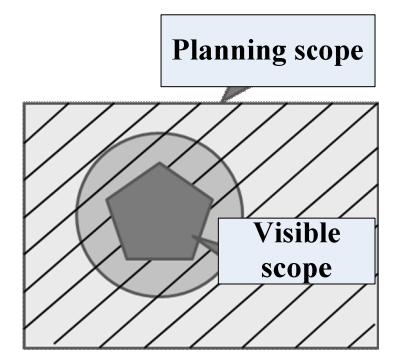

Figure 3. determine scope of invisible area

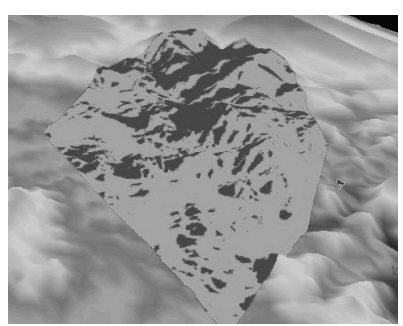

Figure 4. Optimized visible area analysis effect picture 


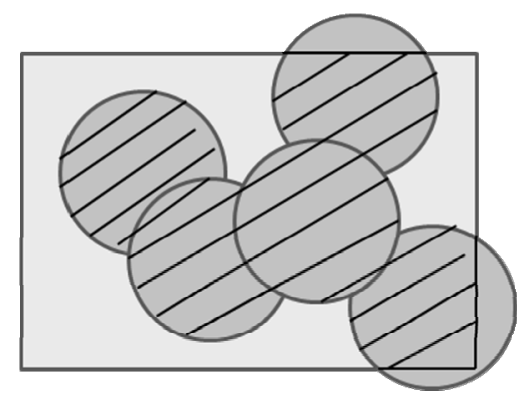

Figure 5. Determine effective area analysis of multi-fire towers

\subsection{Layout Program Statistical Planning Evalu- ation}

Layout program statistical planning evaluation is to compare multi layout programs and select optimized program as the reference for planning construction. First, we should standardize the rules and design access. In this system development, we use relative percentage of blindarea, targeted-area and non-targeted area as the criterion. Blind-area is defined as area which can not be monitored by any fire tower. Non-targeted area is defined as area which can be monitored by only one fire tower. Targeted area is defined as area which can be monitored by two or more than two fire towers. High percentage of targeted area and low percentage of blind-area is the best planning.

To achieve this function, the system has the following four-step processing:

1) get effective analysis scope

Effective scope analysis for several fire towers can be viewed as a collection after superposition of several regions, which shown as the shaded area in figure 5:

2) multi-fire tower visible area analysis
After determining the standard, we can refer the single fire tower visible area analysis method to get related visible and invisible area.

bool flg = VisibleAnalysysByPolygon(m_pGdataBase, 3dDotArr, polygon, visiableId,invisiableId)

In the above-mentioned interface, 3dDotArr represents all the location of fire tower in layout program. VisiableId and invisiableId respectively, are the visible and invisible analyzed results image identification. From the above, we can see that the visible included single-visible fire tower and several-visible fire tower which are distinguished by stored colors.

3) statistical area of the three regions

In the layout program statistical evaluation, one of the most distinctive features is the quantitative evaluation, not only the direct display of visible condition.

The so-called quantitative evaluation is to determine the area of the targeted-area, non-targeted area and blind area. Since analyzed results are stored in raster format, thus the statistic will take full advantage of raster image grid characteristics, and determine the overall percentage of the three regions by "grid numbers".

4) display coverage and statistical results of three regions

System will load the images which get from the last step into the $3 \mathrm{D}$ scene, to get the figure 6 as effect picture. At the same time, the system will display the percentage of targeted-area, non-targeted area and blind area in pie charts.

\section{Conclusion}

As the development of forestry information, GIS and 3D visualization have been the main technology applied in building forestry system. This paper designed and realized the fire tower planning analysis sub-system. This

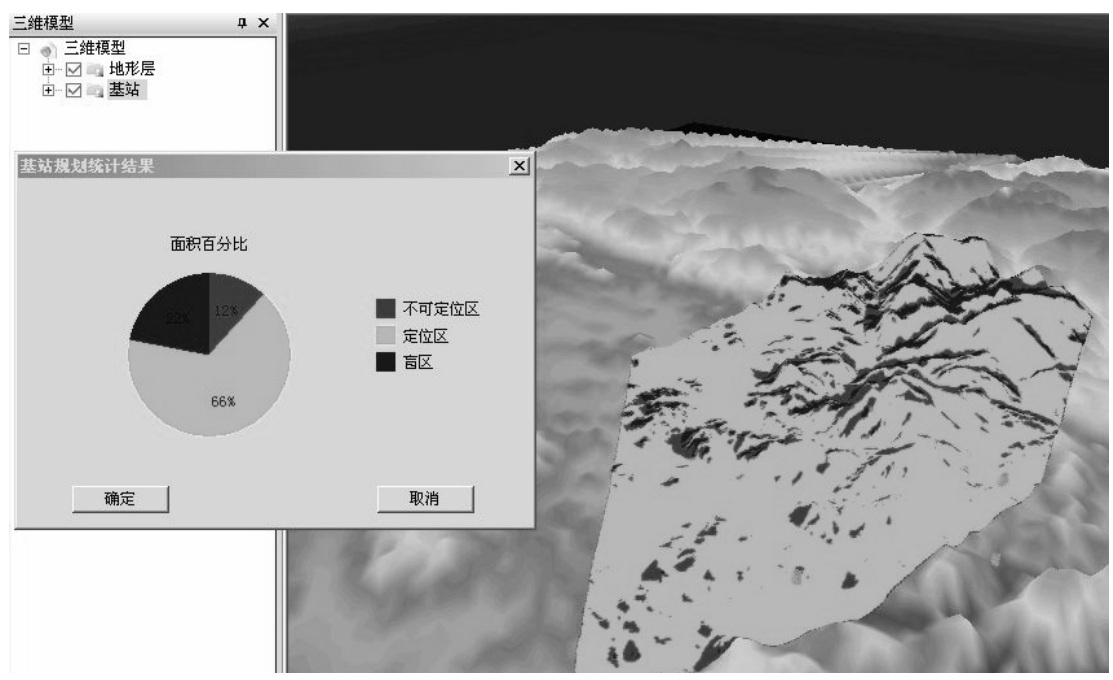

Figure 6. Layout program statistical evaluation effect picture 
system uses GIS and 3D viewable analysis technologies as key technologies. It uses MapGIS platform and 3D platform as developed environment and shows how to realize the fire tower planning analysis sub-system. This system not only benefited forest management departments, for they can scientific planning fire towers, but assistant setting new fire towers location planning, which helps to cut the operational cost and improve forestry management.

\section{REFERENCES}

[1] Guo li na., The analysis of forest fires in visible area in Mohe. Natural sciences journal of Harbin normal univer- sity, 25(1):98-100, 2009.

[2] Guo li na, The application of GIS on observation tower site. Natural sciences journal of Harbin normal University, 200925(4): 102-104,

[3] Chui Xue-ming, Study on Lay out of Fire Tower in the Second Phase Forest Fire Control Project of eight bureau of Lingnan Based on 3D Analyst of ArcGIS. Inner Mongolia Forestry Investigation and Design, 2009.32(5):66-68,

[4] Yu Wendong, He Weijie. Application of Fire Tower in Forest Fire Supervision System, 2008.40(3):28-29.

[5] YU Wen-hua. Design and development of automatic positioning system for forest fire based on GIS Journal of Anhui Agricultural University, 2009, 36(3):493-497. 